\title{
Clinical and Radiographic Peri-Implant Parameters and Whole Salivary Interleukin-1 $\beta$ and Interleukin- 6 Levels among Type-2 Diabetic and Nondiabetic Patients with and without Peri-Implantitis
}

\author{
Mansour Al-Askar ${ }^{\mathrm{a}}$ Sumaiah Ajlan ${ }^{\mathrm{a}}$ Nuha Alomar ${ }^{\mathrm{a}}$ Nasser M. Al-Daghrib \\ ${ }^{a}$ Department of Periodontics and Community Dentistry, College of Dentistry, King Saud University, Riyadh, Kingdom of \\ Saudi Arabia; ${ }^{b}$ Department of Biochemistry, College of Science, King Saud University, Riyadh, Kingdom of Saudi Arabia
}

\section{Significance of the Study}

- Peri-implantitis is a common manifestation in diabetic patients. The aim of this study was to assess peri-implant clinical and radiographic parameters and whole salivary interleukin (IL)-1 $\beta$ and IL-6 levels among type 2 diabetic and nondiabetic patients with and without peri-implantitis. Levels of these markers in nondiabetic patients were higher among patients with peri-implantitis compared to patients without peri-implantitis. Among patients with type 2 diabetes, the severity of these parameters is influenced by the glycemic status rather than by peri-implantitis.

\section{Keywords}

Dental implants · Alveolar bone loss · Gingival bleeding ·

Peri-implantitis · Saliva

\begin{abstract}
Objective: The aim was to assess the peri-implant clinical and radiographic parameters and whole salivary levels of interleukin (IL)- $1 \beta$ and IL- 6 among type 2 diabetic and nondiabetic patients with and without peri-implantitis. Material and Methods: Ninety-one implants were placed in patients without type 2 diabetes mellitus (39 patients with and 52 patients without peri-implantitis; group 1). Eighty implants were placed in patients with diabetes (35 patients with and 45 patients without peri-implantitis; group 2). Peri-implant plaque index, bleeding on probing, probing depth, and marginal bone loss were measured. Unstimulated whole saliva samples were collected and IL-1 $\beta$ and IL- 6 levels were measured using standard techniques. $p<0.05$ was considered statistically significant.
\end{abstract}

Results: In group 1, plaque index ( $p<0.001)$, bleeding on probing $(p<0.001)$, probing depth $(p<0.001)$, and whole salivary IL-1 $\beta(p<0.001)$ and IL-6 $(p<0.001)$ levels were significantly higher in patients with peri-implantitis than in those without peri-implantitis. Plaque index, bleeding on probing, probing depth, and marginal bone loss were comparable among all of the patients in group 2 . Among patients with peri-implantitis, plaque index $(p<0.001)$, bleeding on probing $(p<0.001)$, probing depth $(p<0.001)$, marginal bone loss $(p<$ $0.001)$, and whole salivary IL-1 $\beta(p<0.001)$ and IL-6 $(p<0.001)$ levels were significantly higher in those with diabetes than in those without diabetes. Conclusion: Among individuals without diabetes, peri-implant plaque index, bleeding on probing, probing depth, marginal bone loss, and whole salivary IL-1 $\beta$ and IL-6 levels were higher among patients with peri-implantitis compared to patients without peri-implantitis. Among patients with diabetes, the severity of the measured parameters appears to be influenced by the glycemic status rather than by peri-implantitis.

(C) 2018 The Author(s)

Published by S. Karger AG, Basel 


\section{Introduction}

Implant success and survival rates of up to $100 \%$ have been reported [1]. Despite the predictable success of implants, complications and failures occur in a small percentage of cases $[2,3]$. Peri-implant disease is a chronic inflammatory condition caused by bacterial plaque contamination [4]. It can be classified as peri-implant mucositis, which is reversible inflammation confined to the soft tissue [5], or peri-implantitis, characterized by a progressive inflammatory response that leads to alveolar bone loss [6]. Clinical signs of peri-implantitis that imitate chronic periodontitis include bleeding on probing (BOP), soft-tissue inflammation, increased probing depth (PD), pain, and suppuration [7]. Although bacterial plaque is the primary factor in the etiology of peri-implantitis [8], microbial virulence factors such as lipopolysaccharides enhance the severity of inflammatory responses aided by cytokines released by host immune cells $[9,10]$. Published studies $[11,12]$ have documented strong associations between the release of inflammatory cytokines, such as IL$1 \beta$, IL-6, and IL- 8 , and bone resorption in chronic periodontitis and peri-implantitis.

Poorly controlled type 2 diabetes mellitus (T2DM) has been considered to be a contraindication for implant treatment. The implant failure rate is higher in diabetic patients with adequate metabolic control than in the general population [13]. The implant survival rate in diabetic patients ranges from 88.8 to $97.3 \% 1$ year after placement, and from 85.6 to $94.6 \%$ in functional terms 1 year after prosthesis insertion [14]. The alteration in microvascularization associated with diabetes leads to a diminished immune response, a reduction in bone remodeling processes, and compromise of flap vascularization, thereby delaying healing and providing a gateway for soft-tissue infection $[15,16]$.

Unstimulated whole saliva (UWS) is a complex oral fluid that can be collected easily using noninvasive methods. Studies have shown that a variety of inflammatory biomarkers (e.g., IL-1 $\beta$, IL-6, and immunoglobulins) are present in the UWS of patients with oral and systemic disorders [17-19]. Therefore, the noninvasive assessment of UWS may yield valuable information and can be used to monitor the severity of oral inflammatory conditions, including peri-implantitis $[17,20]$. IL- $1 \beta$ and IL- 6 are the most common inflammatory cytokines that enhance bone resorption by increasing osteoclastic activity [18]. The present study is based on 2 hypotheses. According to the first one, peri-implant clinical (plaque index [PI], $\mathrm{BOP}$, and $\mathrm{PD}$ ) and radiographic (marginal bone loss;
MBL) parameters are worse in patients with diabetes than in those without diabetes; according to the second hypothesis, whole salivary IL- $1 \beta$ and IL- 6 levels are significantly higher among patients with T2DM compared to patients without T2DM. Moreover, it is also hypothesized that the contribution of peri-implantitis in influencing peri-implant clinical and radiographic parameters and whole salivary IL-1b and IL-6 levels is secondary.

The aim of the present retrospective observational study was to assess peri-implant clinical and radiographic parameters and whole salivary IL- $1 \beta$ and IL- 6 levels among type 2 diabetic and nondiabetic patients with and without peri-implantitis.

\section{Materials and Methods}

\section{Ethical Approval}

Ethical approval for this study was obtained from the Ethics Committee of the College of Dentistry Research Center at King Saud University (KSU), Riyadh, Saudi Arabia. This study was performed in accordance with the Helsinki Declaration, as revised in 2013. Written consent was obtained from all of the participants before inclusion.

\section{Exclusion Criteria}

Exclusion criteria were: (1) self-reported systemic disease, such as human immunodeficiency virus infection/acquired immune deficiency syndrome, cardiovascular disorders, epilepsy, hepatic disorders, and renal disorders; (2) antibiotic and/or steroid intake within the previous 3 months; (3) alcohol consumption (individuals consuming alcohol at least once daily for at least 12 months); (4) a history of periodontal treatment within the previous 3 months; and (5) tobacco smoking (individuals smoking at least once daily for the past 12 months [19]) and/or smokeless tobacco consumption (individuals consuming smokeless tobacco products at least once daily for at least 12 months [21]).

\section{Subjects}

Patients with dental implants functioning for an average of 4 years were recruited from the Dental Implant and Periodontics Clinics at the College of Dentistry, KSU. Individuals who reported being systemically healthy were assigned to group 1 , and those who reported being diagnosed with T2DM for at least the past 1 year were assigned to group 2. All of the patients with T2DM were instructed by their health care physician to maintain glycemic levels via dietary control. In group 1, ninety-one implants were placed in patients without T2DM ( 39 patients with and 52 patients without peri-implantitis). In group 2, eighty implants were placed in patients with T2DM (35 patients with and 45 without peri-implantitis).

\section{Assessment of Inter- and Intraexaminer Reliability}

All clinical and radiographic evaluations were performed by 3 trained investigators (N.A, M.A., and S.A.-A.), who were blinded to the study groups. These investigations were performed on 15 patients who were randomly selected from the study groups. Ran- 
Table 1. Characteristics of the study cohort

\begin{tabular}{|c|c|c|c|c|c|c|}
\hline Age, years & $54.8 \pm 9.5$ & $55.4 \pm 11.6$ & $55.3 \pm 10.7$ & $54.5 \pm 5.8$ & $56.8 \pm 6.6$ & $52.8 \pm 8.2$ \\
\hline Family history of diabetes, $\%$ & 35.9 & None & 35.9 & 81.2 & 77.7 & 85.7 \\
\hline Hemoglobin A1c, \% & $4.5 \pm 0.3^{\mathrm{a}}$ & $4.3 \pm 0.4$ & $5.2 \pm 0.1^{\mathrm{b}}$ & $8.7 \pm 1.4$ & $4.7 \pm 0.1^{\mathrm{c}}$ & $9.3 \pm 1.5$ \\
\hline
\end{tabular}

Values are presented as means \pm SD unless otherwise stated. ${ }^{\text {a }}$ Compared to all patients with type 2 diabetes mellitus $(p<0.001)$. ${ }^{\mathrm{b}}$ Compared to type 2 diabetic patients with peri-implantitis $(p<0.001)$. ${ }^{\mathrm{c}}$ Compared to type 2 diabetic patients with peri-implantitis $(p<0.001)$.

domization was done by picking from an opaque bag a paper which stated the assigned participant code number. The overall mean $\kappa$ score for intra- and interexaminer reliability was 0.88 and 0.86 , respectively.

\section{Clinical Peri-Implant Examination}

The investigators blinded to group assignment performed clinical peri-implant examinations. The full-mouth PI was calculated, and BOP and peri-implant PD were measured at 6 sites per implant (mesiobuccal, midbuccal, distobuccal, distolingual/palatal, midlingual/palatal, and mesiolingual/palatal). PD was measured to the nearest millimeter using a graded probe (Hu-Friedy, Chicago, IL, USA). Peri-implantitis was defined as a peri-implant PD $\geq 5 \mathrm{~mm}$ and $\mathrm{MBL} \geq 2 \mathrm{~mm}$ [22].

\section{Marginal Bone Loss}

Bitewing radiographs were taken using a digital tomography machine (KODAK 8000C System; Carestream Dental LLC, Atlanta, GA, USA) and viewed on a calibrated computer screen (SyncMaster digital TV monitor; Samsung, Korea) using a software program (Image Tool 3.0; Department of Dental Diagnostic Science, University of Texas Health Science Center, San Antonio, TX, USA). MBL was measured as the vertical distance from $2 \mathrm{~mm}$ below the crown margin to the most apical part of the marginal bone.

\section{Collection of UWS Samples}

UWS samples were collected between 8:00 a.m. and 9:00 a.m. Participants were asked not to eat or drink for at least $2 \mathrm{~h}$ before sample collection. They were seated comfortably in a dental chair with their head slightly bent forward and instructed to let saliva accumulate in the mouth (without any stimulation) and avoid swallowing, coughing, and moving their lip and tongue during UWS collection for $5 \mathrm{~min}$, after which they were asked to expectorate into a funnel connecting to a gauged measuring cylinder [23]. Immediately after collection, UWS samples were transferred to disposable Eppendorf tubes and placed on ice. The samples were then aliquoted and frozen at $-80^{\circ} \mathrm{C}$. All UWS samples were analyzed within 6 months of collection.

\section{Measurement of IL-1 $\beta$ and IL-6 Levels in UWS}

Levels of unstimulated whole salivary IL- $1 \beta$ and IL- 6 were measured in duplicate using enzyme-linked immunosorbent assays
(ELISA). Human IL-6 (Human Interleukin-6 Quantikine ELISA Kit; R\&D Systems Inc., Minneapolis, MN, USA) and IL-1 $\beta$ (Salivary IL-1 $\beta$ Kit; SALIMETRICS LLC, Carlsbad, CA, USA) kits were used according to the manufacturers' instructions.

\section{Statistical Analysis}

A sample size calculation was performed using nQuery Advisor (version 7.0; Statistical Solutions Ltd., Cork, Ireland) to achieve a type I rate of $\alpha=0.05$ and a power of $85 \%$. Statistical analysis was performed using a software program (SPSS version 18; SPSS Inc., Chicago, IL, USA). Clinical parameters and salivary cytokine concentrations were assessed using one-way analysis of variance. For multiple comparisons, Bonferroni post hoc adjustment was used. $p<0.05$ was considered statistically significant.

\section{Results}

\section{General Characteristics of the Study Population}

All of the participants were male. The mean age of the self-reported nondiabetic individuals with and without peri-implantitis was $55.4 \pm 11.6$ and $55.3 \pm 10.7$ years, respectively. The mean age of the patients with self-reported T2DM with and without peri-implantitis was $56.8 \pm$ 6.6 and $52.8 \pm 8.2$ years, respectively. A family history of diabetes mellitus was reported by 35.9 and $81.2 \%$ of the individuals in groups 1 and 2, respectively.

\section{Hemoglobin A1c Levels}

Mean glycated hemoglobin (HbA1c) levels were significantly higher in group 2 than in group $1(p<0.001)$. In groups 1 and 2, HbA1c levels were significantly higher among individuals with peri-implantitis than in those without peri-implantitis (both $p<0.001$; Table 1 ).

\section{Clinical Peri-Implant Inflammatory Parameters}

Scores of peri-implant PI $(p<0.001)$, BOP $(p<0.001)$, and PD $(p<0.001)$ were significantly higher among indi- 
Table 2. Clinical and radiographic peri-implant parameters among type 2 diabetic and nondiabetic individuals with and without periimplantitis

\begin{tabular}{|c|c|c|c|c|c|c|}
\hline \multirow[t]{2}{*}{ Peri-implant parameter } & \multicolumn{3}{|c|}{ Patients without T2DM } & \multicolumn{3}{|c|}{ Patients with T2DM } \\
\hline & $\begin{array}{l}\text { all individuals } \\
(n=91)\end{array}$ & $\begin{array}{l}\text { without } \\
\text { peri-implantitis } \\
(n=52)\end{array}$ & $\begin{array}{l}\text { with } \\
\text { peri-implantitis } \\
(n=39)\end{array}$ & $\begin{array}{l}\text { all individuals } \\
(n=80)\end{array}$ & $\begin{array}{l}\text { without } \\
\text { peri-implantitis } \\
(n=45)\end{array}$ & $\begin{array}{l}\text { with } \\
\text { peri-implantitis } \\
(n=35)\end{array}$ \\
\hline Plaque index, $\%$ & $31.9 \pm 12.9^{\mathrm{a}}$ & $26.1 \pm 5.4^{b}$ & $38.7 \pm 7.6^{\mathrm{f}}$ & $49.5 \pm 9.4$ & $45.3 \pm 9.7$ & $54.98 \pm 5.4$ \\
\hline Bleeding on probing, $\%$ & $24.3 \pm 8.7^{\mathrm{a}}$ & $25.4 \pm 3.4^{\mathrm{c}}$ & $22.7 \pm 4.5^{\mathrm{g}}$ & $42.8 \pm 7.4$ & $39.5 \pm 4.6$ & $45.3 \pm 6.9$ \\
\hline Peri-implant probing depth, $\mathrm{mm}$ & $3.5 \pm 0.5^{\mathrm{a}}$ & $2.8 \pm 0.1^{\mathrm{d}}$ & $4.5 \pm 0.7^{\mathrm{h}}$ & $5.9 \pm 1.9$ & $5.8 \pm 1$ & $6.7 \pm 1.4$ \\
\hline Marginal bone loss, $\mathrm{mm}$ & $1.4 \pm 0.2^{\mathrm{a}}$ & $0.5 \pm 0.1^{\mathrm{e}}$ & $2.6 \pm 0.3^{\mathrm{i}}$ & $4.9 \pm 1.1$ & $4.5 \pm 0.2$ & $5.1 \pm 0.3$ \\
\hline
\end{tabular}

T2DM, type 2 diabetes mellitus. ${ }^{\text {a }}$ Compared to all individuals with T2DM. ${ }^{b}$ Compared to patients with peri-implantitis but without T2DM. ${ }^{c}$ Compared to patients with peri-implantitis but without T2DM. ${ }^{\mathrm{d}}$ Compared to patients with peri-implantitis but without T2DM. ${ }^{\mathrm{e}}$ Compared to patients with peri-implantitis but without T2DM. ${ }^{\mathrm{f}}$ Compared to patients with peri-implantitis and T2DM. g Compared to patients with peri-implantitis and T2DM. ${ }^{\mathrm{h}}$ Compared to patients with peri-implantitis and T2DM. ${ }^{\mathrm{i}}$ Compared to patients with peri-implantitis and T2DM.

Table 3. Whole salivary IL-1 $\beta$ and IL-6 levels among diabetic and nondiabetic patients with and without peri-implantitis

\begin{tabular}{|c|c|c|c|c|c|c|}
\hline \multirow[t]{2}{*}{ Parameter } & \multicolumn{3}{|c|}{ Patients without T2DM } & \multicolumn{3}{|c|}{ Patients with T2DM } \\
\hline & $\begin{array}{l}\text { all } \\
\text { individuals } \\
(n=91)\end{array}$ & $\begin{array}{l}\text { without } \\
\text { peri-implantitis } \\
(n=52)\end{array}$ & $\begin{array}{l}\text { with } \\
\text { peri-implantitis } \\
(n=39)\end{array}$ & $\begin{array}{l}\text { all } \\
\text { individuals } \\
(n=80)\end{array}$ & $\begin{array}{l}\text { without } \\
\text { peri-implantitis } \\
(n=45)\end{array}$ & $\begin{array}{l}\text { with } \\
\text { peri-implantitis } \\
(n=35)\end{array}$ \\
\hline $\mathrm{IL}-1 \beta, \mathrm{pg} / \mathrm{mL}$ & $978.2 \pm 90.7^{\mathrm{a}}$ & $646.8 \pm 60.1^{b}$ & $1,153.54 \pm 118.5^{\mathrm{c}}$ & $2,171.6 \pm 171.1$ & $2,023.4 \pm 98.4$ & $3,075.83 \pm 723.8$ \\
\hline $\mathrm{IL}-6, \mathrm{pg} / \mathrm{mL}$ & $42.28 \pm 8.4^{\mathrm{a}}$ & $39.7 \pm 4.4^{\mathrm{c}}$ & $45.62 \pm 5.1$ & $80.2 \pm 12.5$ & $76.73 \pm 4.2$ & $132.06 \pm 9.5$ \\
\hline
\end{tabular}

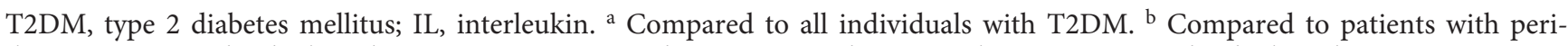
implantitis among individuals without T2DM. ${ }^{c}$ Compared to patients with peri-implantitis among individuals without T2DM.

viduals in group 2 compared to group 1 . In group 1 , periimplant PI $(p<0.001)$, BOP $(p<0.001)$, and PD $(p<$ 0.001 ) were significantly higher among individuals with peri-implantitis compared to individuals without periimplantitis. In group 2, there was no statistically significant difference in the scores of peri-implant PI, BOP, and $\mathrm{PD}$ among individuals with and without peri-implantitis. Among individuals with peri-implantitis, scores of periimplant PI $(p<0.001)$, BOP $(p<0.001)$, and PD $(p<$ 0.001 ) were significantly higher among individuals in group 2 compared to group 1 (Table 2).

\section{Marginal Bone Loss}

In group 1 , the peri-implant MBL $(p<0.001)$ was statistically significantly higher among patients with periimplantitis as compared to patients without it. In group 2 , there was no statistically significant difference in peri- implant MBL among individuals with and without periimplantitis. Overall, peri-implant MBL $(p<0.001)$ was significantly higher among individuals in group $2 \mathrm{com}-$ pared to group 1 (Table 2).

\section{Levels of Whole Salivary IL-1 $\beta$ and IL-6}

Levels of whole salivary IL-1 $\beta(p<0.001)$ and IL-6 $(p<0.001)$ were significantly higher in group 2 compared to group 1 . In group 1 , whole salivary IL-1 $\beta(p<0.001)$ and IL-6 $(p<0.001)$ levels were significantly higher among patients with peri-implantitis compared to patients without peri-implantitis. In group 2, there was no statistically significant difference in levels of whole salivary IL- $1 \beta$ and IL-6 among patients with and without peri-implantitis. Overall, levels of whole salivary IL-1 $\beta(p<0.001)$ and IL-6 $(p<0.001)$ were significantly higher among patients in group 2 compared to group 1 (Table 3 ). 


\section{Discussion}

The present retrospective observational study is based on the hypotheses that: (1) peri-implant clinical (PI, BOP, and $\mathrm{PD}$ ) and radiographic (MBL) inflammatory parameters are worse and levels of IL- $1 \beta$ and IL- 6 in the UWS are higher in patients with T2DM (group 2) compared to patients without T2DM (group 1), and (2) the contribution of peri-implantitis in aggravating the clinical and radiographic inflammatory parameters and increasing the levels of whole salivary proinflammatory cytokines IL- $1 \beta$ and IL- 6 is secondary. The present results are in accordance with the proposed hypotheses.

Various explanations can be proposed in this context. A critical finding of the present study is that all of the diabetic individuals had poorly controlled T2DM, that is, their mean HbA1c levels were $>8 \%$ [24]. A state of chronic hyperglycemia has been associated with increased formation of advanced glycation end produts (AGE) and their accumulation in tissues including those of the oral cavity [25]. It has been reported that increased interactions between AGE and their receptors are associated with an increased severity of tissue inflammation and raised levels of proinflammatory cytokines in saliva, follicular fluid, and serum [26-28]. As all of the patients with T2DM included in the present study had HbA1c levels higher than $8 \%$, it is likely that there was an increased expression of AGE and interactions between AGE and receptor of AGE among individuals in group 2 (regardless of the presence or absence of peri-implantitis) compared to group 1. This is a possible explanation for the poorer peri-implant clinical (PI, BOP, and PD) and radiographic (MBL) parameters and increased levels of IL- $1 \beta$ and IL- 6 in the UWS of diabetic patients with and without peri-implantitis. With reference to individuals in group 1, the state of local inflammation seems to control the peri-implant clinical, radiographic, and inflammatory parameters and whole salivary cytokine levels. These results suggest that among patients without T2DM, routine dental visits and maintenance of regular oral hygiene may play a role in the prevention and treatment of peri-implant diseases. However, among T2DM patients, glycemic maintenance in addition to routine dental visits and regular oral hygiene maintenance are recommended.

UWS is a complex oral fluid that can also be used to assess the prognosis of oral diseases, such as periodontitis [29]. In the present study, levels of IL- $1 \beta$ and IL- 6 were statistically significantly higher among individuals in group 1 with peri-implantitis compared to those without peri-implantitis. It has been reported that IL- $1 \beta$ and IL-6 enhance bone resorption by increasing osteoclastic activity. This may be an explanation for the increased periimplant MBL among individuals with peri-implantitis compared to individuals without peri-implantitis in group 1. These results indicate that assessment of proinflammatory cytokines in the UWS may also be useful in assessing the progression of peri-implantitis and not merely periodontitis. However, further studies are needed to test this hypothesis.

A limitation of the present study is that all of the individuals who agreed to participate were male despite the inclusion of females in the recruitment effort. Hormonal changes and postmenopausal status are known to influence periodontal health status. Thus, the expression of periodontal and peri-implant disease activity may differ between men and postmenopausal women with T2DM. In addition, the present study did not include tobacco consumers. Habitual tobacco smoking and use of smokeless tobacco products are known to be significant risk factors for periodontal disease [30]. Thus, periodontal and peri-implant diseases may be more severe in tobacco consumers than in those who do not consume tobacco. Further studies are needed to investigate these issues.

\section{Conclusion}

Among nondiabetic individuals, peri-implant PI, $\mathrm{BOP}, \mathrm{PD}$, and MBL and whole salivary IL- $1 \beta$ and IL-6 levels are higher among patients with peri-implantitis compared to patients without peri-implantitis. Among patients with T2DM, the severity of the measured parameters is influenced by the glycemic status rather than by peri-implantitis.

\section{Acknowledgment}

This project was supported financially by the Vice Deanship of Scientific Research, King Saud University, Riyadh, Kingdom of Saudi Arabia. The authors also thank the Prince Mutaib Chair for Biomarkers of Osteoporosis, College of Science, King Saud University, for supporting the analysis of inflammatory markers.
Clinical Peri-Implant Parameters and

Peri-Implantitis in Type 2 Diabetes
Med Princ Pract 2018;27:133-138

DOI: $10.1159 / 000488032$ 


\section{References}

1 Winkler S: Extraordinary implant failure. J Oral Implantol 2010;36:391-400.

2 Park JH, Kim YS, Ryu JJ, et al: Cumulative survival rate and associated risk factors of Implantium implants: a 10-year retrospective clinical study. J Adv Prosthodont 2017;9:195199.

3 Sennerby L: Dental implants: matters of course and controversies. Periodontol 2000; 2008;47:9-14.

4 Peri-implant mucositis and peri-implantitis: a current understanding of their diagnoses and clinical implications. J Periodontol 2013; 84:436-443.

5 Zitzmann NU, Berglundh T, Marinello CP, et al: Experimental peri-implant mucositis in man. J Clin Periodontol 2001;28:517-523.

6 Sanz M, Chapple IL; Working Group 4 of the VIII European Workshop of Periodontology: Clinical research on peri-implant diseases: consensus report of Working Group 4. J Clin Periodontol 2012;39(suppl 12):202-206.

7 Finne K, Rompen E, Toljanic J: Prospective multicenter study of marginal bone level and soft tissue health of a one-piece implant after two years. J Prosthet Dent 2007;97:S79-S85.

8 Liskmann S, Vihalemm T, Salum O, et al: Correlations between clinical parameters and interleukin-6 and interleukin-10 levels in saliva from totally edentulous patients with peri-implant disease. Int J Oral Maxillofac Implants 2006;21:543-550.

9 Deo V, Bhongade ML: Pathogenesis of periodontitis: role of cytokines in host response. Dent Today 2010;29:60-62.

10 Murata M, Tatsumi J, Kato Y, et al: Osteocalcin, deoxypyridinoline and interleukin-1beta in peri-implant crevicular fluid of patients with peri-implantitis. Clin Oral Implants Res 2002;13:637-643.

11 Kornman KS, Page RC, Tonetti MS: The host response to the microbial challenge in periodontitis: assembling the players. Periodontol 2000 1997; 14:33-53.
12 Panagakos FS, Aboyoussef H, Dondero R, et al: Detection and measurement of inflammatory cytokines in implant crevicular fluid: a pilot study. Int J Oral Maxillofac Implants 1996;11:794-799.

13 Morris HF, Ochi S, Winkler S: Implant survival in patients with type 2 diabetes: placement to 36 months. Ann Periodontol 2000;5: 157-165.

14 Fiorellini JP, Nevins ML, Norkin A, et al: The effect of insulin therapy on osseointegration in a diabetic rat model. Clin Oral Implants Res 1999; 10:362-368

15 Olson JW, Shernoff AF, Tarlow JL, et al: Dental endosseous implant assessments in a type 2 diabetic population: a prospective study. Int J Oral Maxillofac Implants 2000;15:811-818.

16 Farzad P, Andersson L, Nyberg J: Dental implant treatment in diabetic patients. Implant Dent 2002;11:262-267.

17 Ataoglu H, Alptekin NO, Haliloglu S, et al: Interleukin-1beta, tumor necrosis factor-alpha levels and neutrophil elastase activity in peri-implant crevicular fluid. Clin Oral Implants Res 2002;13:470-476.

18 Stashenko P, Fujiyoshi P, Obernesser MS, et al: Levels of interleukin 1 beta in tissue from sites of active periodontal disease. J Clin Periodontol 1991;18:548-554

19 Javed F, Nasstrom K, Benchimol D, et al: Comparison of periodontal and socioeconomic status between subjects with type 2 diabetes mellitus and non-diabetic controls. J Periodontol 2007;78:2112-2119.

20 Fonseca FJ, Moraes M Jr, Lourenco EJ, et al: Cytokines expression in saliva and peri-implant crevicular fluid of patients with peri-implant disease. Clin Oral Implants Res 2014; 25:e68-e72.

21 Abduljabbar T, Hussain M, Adnan T, et al: Comparison of oral Candida species prevalence and carriage among gutka-chewers and betel-quid chewers. J Pak Med Assoc 2017;67: 350-354.
22 Nguyen-Hieu T, Borghetti A, Aboudharam G: Peri-implantitis: from diagnosis to therapeutics. J Investig Clin Dent 2012;3:79-94.

23 Fiyaz M, Ramesh A, Ramalingam K, et al: Association of salivary calcium, phosphate, $\mathrm{pH}$ and flow rate on oral health: a study on 90 subjects. J Indian Soc Periodontol 2013;17: 454-460.

24 American Diabetes Association: Classification and Diagnosis of Diabetes: Standards of Medical Care in Diabetes-2018. Part 2. Diabetes Care 2018;41:S13-S27.

25 Schmidt AM, Weidman E, Lalla E, et al: Advanced glycation endproducts (AGEs) induce oxidant stress in the gingiva: a potential mechanism underlying accelerated periodontal disease associated with diabetes. J Periodontal Res 1996;31:508-515.

26 Yoon MS, Jankowski V, Montag S, et al: Characterisation of advanced glycation endproducts in saliva from patients with diabetes mellitus. Biochem Biophys Res Commun 2004; 323:377-381.

27 Wang B, Hao M, Yang Q, et al: Follicular fluid soluble receptor for advanced glycation end products (sRAGE): a potential protective role in polycystic ovary syndrome. J Assist Reprod Genet 2016;33:959-965.

28 Yilmaz Y, Yonal O, Eren F, et al: Serum levels of soluble receptor for advanced glycation endproducts (sRAGE) are higher in ulcerative colitis and correlate with disease activity. J Crohns Colitis 2011;5:402-406.

29 Ji S, Choi Y: Point-of-care diagnosis of periodontitis using saliva: technically feasible but still a challenge. Front Cell Infect Microbiol 2015;5:65.

30 Al-Askar M, Abduljabbar T, Vohra F, et al: Comparison of self-perceived oral symptoms and periodontal parameters among habitual shamma-chewers, gutka-chewers, and nonchewers. Quintessence Int 2017;48:251-260. 